\title{
A fast and robust ellipse detector based on top-down least-square fitting
}

\author{
Yongtao Wang \\ wyt@pku.edu.cn \\ Zheqi He \\ philokeys@gmail.com \\ Xicheng Liu \\ liuxicheng@pku.edu.cn \\ Zhi Tang \\ tangzhi@pku.edu.cn \\ Luyuan Li \\ liluyuan@pku.edu.cn
}

\author{
Institute of Computer Science \& Technology \\ Peking University \\ Beijing, China
}

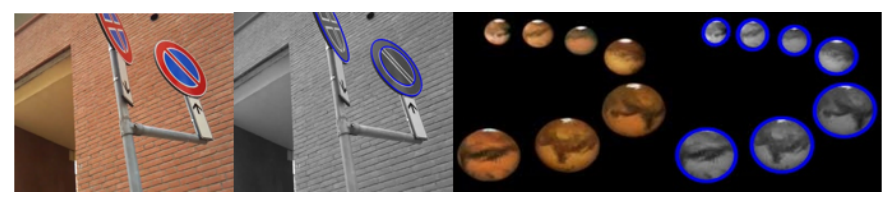

Figure 1: Detecting ellipses from images

Detecting ellipses efficiently and accurately in digital images is an fundamental problem to the field of pattern recognition and computer vision. Ellipse detection can be used in many applications. The existing algorithms such as [4] often use a bottom-up strategy to combine edge points or elliptical arcs into ellipses, which limits their robustness. In this paper, we propose a novel algorithm which can efficiently and accurately detect ellipses in digital images with a novel top-down scheme. The main idea of the proposed algorithm is to exploit a novel top-down fitting strategy to combine edge points into ellipses and use integral chain to speed up the fitting process. The proposed method is very efficient, which is faster than the most efficient method [1] which has been reported. Our experimental results also demonstrate that our algorithm is more robust than the state-of-the-art methods including [1] and ELSD [4].As shown in Fig. 2, our method consists of four major steps.

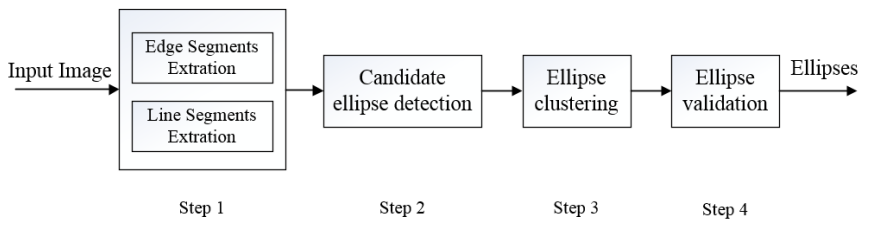

Figure 2: Workflow of the proposed ellipse detection method

In the first step, we extract edge segments from the input image and then obtain line segments within each edge segment. We directly use the edge segment extraction method and the line segment detection method proposed in $[2,3]$ respectively, which are very computational efficient. Secondly, we detect candidate ellipses from each edge segment by the topdown analysis. In this step, we use the 1-D case(which is called "integral chain" hereafter) of integral image technique [5] to speed up the proposed ellipse detection algorithm.Specifically, for a sequence $x_{1}, x_{2}, \ldots, x_{n}$, the corresponding integral chain is:

$$
\operatorname{IntChain}(k)=\sum_{i=1}^{k} x_{i}, k=1, . . n .
$$

The recurrent formulas for computing the integral chain are:

$$
\operatorname{IntChain}(1)=x_{1}, \quad \operatorname{IntChain}(k)=\operatorname{IntChain}(k-1)+x_{k}, \quad k>1 .
$$

Within the integral chain, the sum of any sub-sequence can be efficiently obtained by

$$
\sum_{i=1}^{k} x_{i}=\operatorname{IntChain}(k), \sum_{i=s}^{k} x_{i}=\operatorname{IntChain}(k)-\operatorname{IntChain}(s-1) .
$$

After that, we cluster candidate ellipses to merge and remove the fractional detections, because a complete ellipse may be split into two or more candidate ellipses due to the incomplete edge segment extraction results. Finally, we eliminate false alarms using NFA validation. In detail, for an ellipse with $l$ independent edge points, there are $k$ of these points having their gradient directions aligned with the direction of this ellipse, where aligned means that a point $p_{i}$ is at the direction orthogonal to the tangent of the ellipse on $p_{i}$. The expectation of such kind of ellipse, so-called "number of false alarms"(NFA), should be less than $\varphi$, that is:

$$
N F A(l, k)=N_{t} \sum_{i=k}^{l}\left(\begin{array}{l}
l \\
i
\end{array}\right) p^{i}(1-p)^{l-i}<\varphi,
$$

where $N_{t}$ is the number of all possible ellipses; $\varphi$ is set to 1 and the precision of the direction alignment $p$ is set to $1 / 8$ as mentioned in [4]. We calculate the gradient direction of each edge point in the original image with Sobel operator(the aperture size is 3).

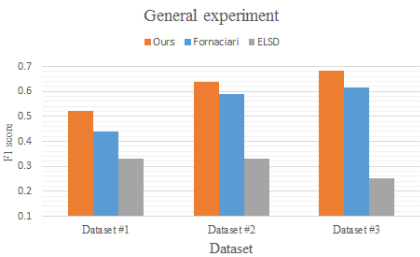

(a)

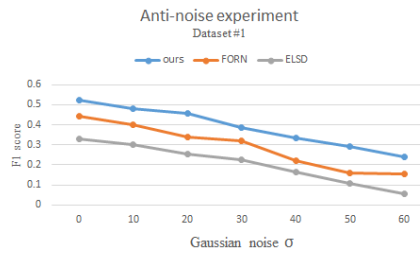

(b)
Figure 3: (a) F1 scores of general experiment; (b) F1 scores of anti-noise experiment on Dateset \#1

We make experiments on three public real image datasets. We compare the performance of our method with two state-of-the-art ellipse detection methods: (1) Fornaciari et al.'s method (FORN) which is proposed in [1], and (2) ELSD [4] which is proposed by Puatruaucean et al. Fig. 3 illustrate the result on the three datasets.

The general experiments indicate the proposed method achieves better performance than the other two methods: it runs faster while achieving the highest F1 score. The anti-noise experiments evaluate robustness to noise of these methods, we add Gaussian noise with different level (the variance $\sigma^{2}=\left\{0,10^{2}, 20^{2}, 30^{2}, 40^{2}, 50^{2}, 60^{2}\right\}$ ) to images in the three datasets. The result indicates that our method is more robust than the two other methods.

[1] Michele Fornaciari, Andrea Prati, and Rita Cucchiara. A fast and effective ellipse detector for embedded vision applications. Pattern Recognition, 47(11):3693-3708, 2014.

[2] Dong Liu, Yongtao Wang, Zhi Tang, and Xiaoqing Lu. A robust circle detection algorithm based on top-down least-square fitting analysis. Computers \& Electrical Engineering, 40(4):1415-1428, 2014.

[3] Dong Liu, Yongtao Wang, Zhi Tang, and Xiaoqing Lu. A robust and fast line segment detector based on top-down smaller eigenvalue analysis. In Fifth International Conference on Graphic and Image Processing, pages 906916-906916. International Society for Optics and Photonics, 2014.

[4] Viorica Pătrăucean, Pierre Gurdjos, and Rafael Grompone Von Gioi. A parameterless line segment and elliptical arc detector with enhanced ellipse fitting. In Computer Vision-ECCV 2012, pages 572585. Springer, 2012.

[5] Paul Viola and Michael J Jones. Robust real-time face detection. International journal of computer vision, 57(2):137-154, 2004. 